\section{An Electrophoresis Apparatus for analysing Several Sera Simultaneously}

A SYSTEMATIC study, such as we contemplate, on the influence of disease on the electrophoresis pattern of serum, requires observations on large numbers of sera. Such an investigation is laborious and timeconsuming when the available techniques and apparatus are utilized, because only a very limited number of experiments can be completed in one day. We have, therefore, devised a method whereby two electrophoretic analyses can be made simultaneously using the Tiselius apparatus with a standard central section.

The apparatus is illustrated in Fig. 1. The electrode vessels are connected on to 'Perspex' adaptors ( $A$ and $B$ ) and the standard central section of the electrophoresis cell held between them. Prior to assembly, the ground surfaces of the 'Perspex' adaptors as well as those of the electrode cell are well greased, and thin 'Cellophane' membranes are then clamped between the sections in the position indicated $\left(M_{1}\right.$ and $\left.M_{2}\right)$. The protein solutions to be analysed are held between the membranes, and the rest of the apparatus filled with buffer and saturated potassium chloride in the usual way. The dimensions of the adaptors are such that they fit the Hilger electrophoresis bath and cell holder.

A $p H$ for electrophoresis must be selected where all the components present migrate towards the bottom membrane. In the case of serum, this is achieved at $p H$ values above neutrality.

In Fig. 2 are shown electrophoresis diagrams of two samples of the same bovine serum obtained simultaneously using the two limbs of the standard electrophoresis cell. On inspection of the diagrams it will be noticed that they are identical. The reason for the markedly enhanced $\delta$-effect visible at the beginning of the electrophoresis diagram is, as yet, unexplained. In the diagram of the same serum obtained with the standard apparatus, the $\delta$-effect was small.

Electrosmotic effects do exist; but apparently they have no effect on the electrophoresis diagram. Mobility measurements calculated from electro-

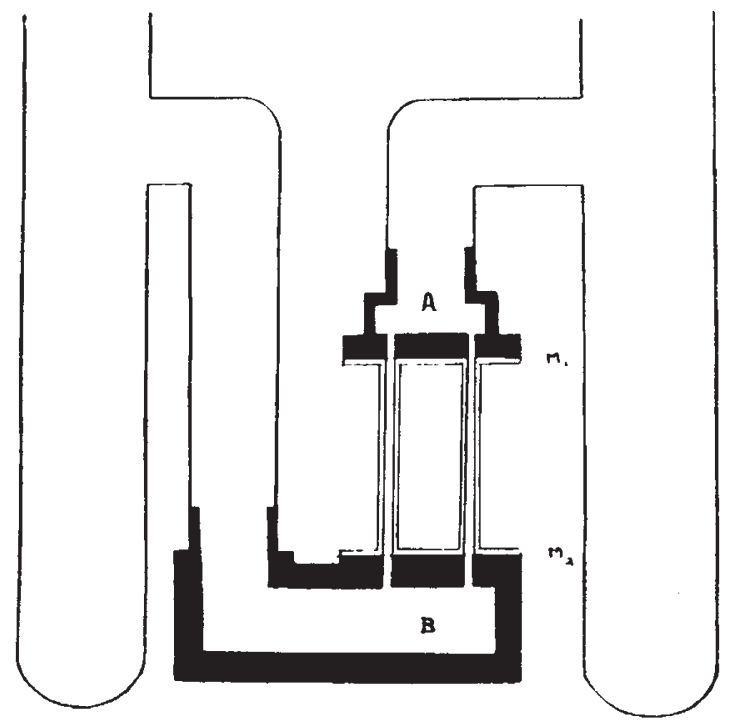

Fig. 1. Assembled electrophoresis cell. Shaded parts ( $A$ and $B$ ) are made of 'Perspex'. $M_{1}$ and $M_{2}$ are 'Cellophane' membranes

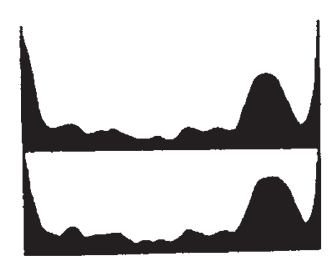

Fig. 2

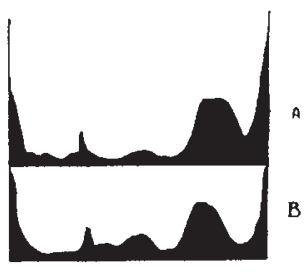

Fig. 3
Fig. 2. Electrophoresis diagrams of two samples of the same bovine serum obtained simultaneously

Fig. 3. Electrophoresis diagrams of normal serum $(A)$ and serum from a chronic nephritic $(B)$. They were obtained simultaneously by the use of the modifled cell

phoresis diagrams obtained in the modified apparatus described are likely to be effected by the electrosmotic effects, but the samples studied simultaneously are comparable.

The technique described points the way to a means of mass electrophoresis of sera, as there is no theoretical limit to the number of compartments which can be built into the analytical section of the cell.

A further advantage of this type of cell is that exact comparisons can be made between sera obtained from different sources. This advantage is clearly brought out in Fig. 3, which shows the difference between the serum from a normal and a chronic nephritic subject. The nephritic serum albumin has a mobility slightly less than that of the normal albumin. The $\alpha$-globulin has the same mobility in both sera, and the $\beta$-globulin in the normal serum has, in turn, a lower migration-rate than the corre. sponding globulin in nephritic serum.

This investigation was assisted by grants from the Council for Scientific and Industrial Research and the Nkane Kitwe Poliomyelitis Research Fund.

Virus Research Unit,

A. Pols on

Department of Pathology,

University of Cape Town. July 5.

\section{Isolation of a Crystalline Disaccharide, Hyalobiuronic Acid, from Hyaluronic Acid}

THE structure of hyaluronic acid, an important constituent of the 'ground substance', has been the subject of intensive study in recent years. Applications of conventional methylation and periodate consumption methods to the intact polysaccharide ${ }^{1,2}$ have indicated that such methods will not yield substantial evidence of structure. The isolation and clarification of structure of crystalline oligosaccharides derived from hyaluronic acid is the most rigorous method of studying the structural details of this physiologically important substance. Such welldefined compounds are also required as models for establishing the specificity of the enzyme systems acting on hyaluronic acid.

The present communication is concerned with the isolation and characterization of a crystalline aldobiuronic acid from sodium hyaluronate in relatively high yield, following degradation by a combination of enzymic and mild acid hydrolysis. Sodium hyaluronate (analysis ${ }^{3}$ : nitrogen, $3 \cdot 78$; uronic acid, 44.0 ; hexosamine, 38.8 ; sulphate, less than 0.5 ) was hydrolysed with purified testicular hyaluronidase 